\title{
A Stepladder Approach to a Tokamak Fusion Power Plant
}

\author{
${ }^{1} \mathrm{H}$. Zohm, ${ }^{1,2} \mathrm{~F}$. Träuble, ${ }^{3} \mathrm{~W}$. Biel, ${ }^{1} \mathrm{E}$. Fable, ${ }^{4} \mathrm{R}$. Kemp, ${ }^{4} \mathrm{H}$. Lux, ${ }^{1} \mathrm{M}$. Siccinio, ${ }^{1,5} \mathrm{R}$. Wenninger \\ ${ }^{1}$ Max-Planck-Institut für Plasmaphysik, Boltzmannstr. 2, D-85748 Garching, Germany \\ ${ }^{2}$ Fakultät für Physik, Ludwig-Maximilians-Universität, D-80799 München, Germany \\ ${ }^{3}$ Institut für Energie- und Klimaforschung, Forschungszentrum Jülich, D-52428 Jülich, Germany \\ ${ }^{4}$ Culham Centre for Fusion Energy, Culham, Abingdon OX14 3EA, United Kingdom \\ ${ }^{5}$ EUROfusion Programme Management Unit, D-85748 Garching, Boltzmannstr. 2, Germany
}

\begin{abstract}
We present an approach to design in a consistent way a stepladder connecting ITER, DEMO and an FPP, starting from an attractive FPP and then locating DEMO such that main similarity parameters for the core scenario are constant. The approach presented suggests how to use ITER such that DEMO can be extrapolated with maximum confidence and a development path for plasma scenarios in ITER follows from our approach, moving from low $\beta_{\mathrm{N}}$ and $\mathrm{q}$ typical for the present $\mathrm{Q}=10$ scenario to higher values needed for steady state. A numerical example is given, indicative of the feasibility of the approach, and it is backed up by more detailed 1.5-D calculation using the ASTRA code. We note that ideal MHD stability analysis of the DEMO operating point indicates that it is located between the no-wall and the ideal wall $\beta$-limit, which may require active stabilization. The DEMO design could also be a pulsed fallback solution should a stationary operation turn out to be impossible.
\end{abstract}

\section{1.) Introduction}

In the EU 'fast track' strategy to a Fusion Power Plant (FPP), DEMO is the single step between ITER and an FPP. The high level goals of the EU DEMO have been described as [1]

- demonstrate a workable solution for all physics and technology questions, demonstrating the favourable safety and environmental properties

- demonstrate large scale net electricity production with self-sufficient fuel cycle

- prove high reliability and availability over a reasonable time span

- allow to assess the economic prospects of an FPP.

It is however not obvious how to derive from these goals a DEMO design point. In fact, the present EU approach examines a variety of options which, depending on the assumption of available physics and technology base at the time of DEMO construction, range from a conservative pulsed device largely based on the ITER $Q=10$ scenario ('DEMO1') to a steady-state machine with more aggressive physics and technology assumptions ('DEMO2') [2]. One central point in using DEMO in the fast track strategy is however, to allow safe extrapolation to an FPP.

In this contribution, we argue that for the plasma scenario, it is inconceivable that there will be a large development step from DEMO to the FPP, since there is no machine other than DEMO itself to qualify it (even if 'satellite' devices exist, the credibility of improvements made in these will not be high enough to be incorporated into a commercial FPP unless they have been demonstrated in the DEMO environment). In a similar manner, ITER should prepare the DEMO scenario since it will be the largest device of its kind and the one that allows to study $\alpha$-particle dynamics and self-heating under conditions closest to DEMO. This is important since with the transition to self-heated plasmas, self-organisation and its controllability 
will become a critical element. While satellite devices such as JT-60SA will play an important role in developing plasma scenarios, it is hardly believable that a scenario developed there will be used for DEMO, but not tested in ITER.

It follows that there should be a stepladder approach ITER-DEMO-FPP that keeps the plasma scenarios as close as possible such that DEMO effectively becomes a technology demonstrator and not a plasma physics experiment, consistent with the original fast track approach. We will first outline a 0-d approach that keeps important dimensionless plasma parameters similar and essentially varies machine size and with it the fusion power and electricity output. Different from the present EU approach that designs DEMO in a forward step from ITER, we use a procedure where the FPP is used as a guideline and then DEMO derived from it. For ITER, this approach means defining an operational point that is prototypical of an FPP, which is not necessarily the present $Q=10$ point. This is explained in Section 2, where also the simple 0-D model is outlined that is used for scoping studies of a stepladder. Section 3 gives a numerical example of such a stepladder, based on the 0-D approach. This example is then studied in Section 4 using the 1.5-D transport code ASTRA to validate the approach accounting for more detailed profile effects. A discussion and conclusions are given in Section 5.

We note here that our approach is targeted at using a consistent set of physics assumptions, while constraints arising from technology are not considered explicitly and their inclusion may set boundaries or open up new possibilities. While beyond the scope of this contribution, a more detailed analysis of the technology constraints should be done in a future step. However, we note that the technology assumptions for our DEMO and FPP steps are in the range of the used for the present EU DEMO [2] and the previous EU Power Plant Conceptual Design Studies ('model A/B' or 'model B' from reference [3]), respectively, so have a firm basis for principal design choices like the blanket or the toroidal field coil design.

\section{2.) 0-D approach for a family of tokamak devices in the burning plasma regime ('stepladder')}

In the preceding section, we have argued that ITER, DEMO and FPP should form a stepladder of tokamak devices using the same plasma scenario. Given the different Q-values of the machines, this requires a choice of appropriate similarity parameters. Here, we will outline an approach that uses a set of 0 -d similarity parameters to define this plasma scenario. We chose to describe the core plasma scenario in terms of dimensionless quantities $\beta_{N}, q_{95}, H$ and $f_{G W}$. The definition and interrelations of these parameters are based on [4] and the full set of equations is given for reference in the appendix. In addition, we chose a constant absolute value of the density since this will be a key parameter for the divertor performance. Different from the usual dimensionless plasma parameter argument, this means that $\rho^{*}$ and $v^{*}$ will vary throughout the stepladder, but we assume the three machines to be big enough, i.e. $\rho^{*}$ low enough [5], that gyro-Bohm scaling, known to be independent of the device size, holds, and $v^{*}$ so low that collisionless physics prevails in all three devices. Hence, while the scalings used may depend on $\rho^{*}$, or $v^{*}$, they will not exhibit size-dependent transitions between regimes where different physics is dominating.

This leaves open the choice of machine parameters $A, R$ and $B$. Since the performance of a plasma scenario can also depend on $A$, we chose to also keep this constant, but it could be relaxed somewhat if a different aspect ratio than that of ITER will prove to have significant advantage. As pointed out in the appendix, we also fix the plasma shape to that of ITER, assuming an elongation of $\kappa=1.7$ and a triangularity of $\delta=0.33$ on

1 For the devices presented in Section 3 and 4 , the volume averaged values of $1 / \rho^{*}$ and $v^{*}$ are $(536,701,735)$ and $(0.012,0.0075,0.005)$ for (ITER, DEMO, FPP). 
the last closed flux surface, resulting in a shaping factor of $S=2.6$ (see Eqn. (A2)). Of course, this choice can be adapted if needed in future studies. As an example, an increase in elongation will be very favorable for the bootstrap fraction since it allows to run higher $q_{95}$ at the same current.

The postulate of constant $f_{G W}$ and absolute density gives a prescription how to vary $B$ and $R$ from machine to machine. Using (A7) and (A10), we obtain

$$
n=f_{G W} \frac{c_{7}}{\pi} \frac{B}{R q_{95}}
$$

which, because of the assumption of constant $q$, leads to $B / R=$ const. Then, at constant $q, \beta_{N}$ and $A$ in (A1), this constraint leads to a scaling of

$$
P_{f u s} \propto R^{7} \propto B^{7}
$$

which means that both $B$ and $R$ will increase according to $P_{f u s}^{1 / 7}$ in the stepladder.

We note that from (A3), it can be seen that increasing both $B$ and $R$ will also strongly increase $Q$, so that achieving ignition in itself will not be a problem in this approach. We also note that the power needed to drive the current in steady state varies surprisingly weakly through the stepladder since Eqns. (A4) to (A11) can be combined to give

$$
P_{C D}=\frac{c_{8} c_{3} c_{5} c_{7}^{3}}{\pi^{2}} \frac{B}{q_{95}^{2}} \frac{f_{G W}^{2}}{\beta_{N} A}\left(5+\frac{\pi^{2} A^{2} q_{95}^{2}}{C_{6} C_{7}^{2} f_{G W}^{2} B^{2}}\left(\frac{P_{f u s}}{5 R}+\frac{P_{C D}}{R}-\frac{P_{s e p}}{R}\right)\right)\left(1-C_{9} \sqrt{A} q_{95} \frac{c_{4}}{c_{3}} \beta_{N}\right)
$$

which in our approach only depends linearly on B (see Eqns. (A8) and (A9)) in the regime where $Z_{\text {eff }}$ is significantly smaller than 5 (so that the second term in the first bracket does not matter). Note that the right hand side of Eqn. (3) still contains $P_{C D}$. It can be easily rearranged to solve for $P_{C D}$, but has been kept like this for the clarity of physics discussion. It follows that in this regime, $\mathrm{P}_{C D}$ increases linearly with $\mathrm{B}$, i.e. only with $\mathrm{P}_{\text {fus }}{ }^{1 / 7}$, so that going from DEMO to an FPP will lead to a significant decrease in recirculating power, but since we do not change the physics scenarios and its assumptions, this should be a highly credible step, in line with the high-level goals outlined in Section 1. We note here that while $Q$ calculated from (A3) can take on very high values for FPP, it will finally be determined by the current drive requirements, approaching $Q=P_{f u s} / P_{C D}$.

Concerning the exhaust problem, we will assume that DEMO and an FPP use the ITER divertor solution, i.e. a single null poloidal divertor operating in detached mode. As figure of merit for divertor performance, we use the tolerable power flowing into a ring of surface $2 \pi R \lambda_{q}$, where $\lambda_{q}$ is the power decay length in the midplane outside, scaling mainly with poloidal gyroradius [6], i.e. $\lambda_{q} \propto q_{95} A / B$ if we neglect the temperature variation (due to the strong temperature dependence of power flux conducted in the SOL, the separatrix temperature is not expected to vary strongly within the stepladder). Our exhaust criterion hence becomes

$$
\frac{P_{\text {sep }} B}{q_{95} A R}<\left(\frac{P_{\text {sep }} B}{q_{95} A R}\right)_{\text {ITER }}
$$

where $P_{\text {sep }}$ is the power crossing the separatrix after subtracting the radiation as given in the Appendix (A8) and the index ITER refers to the $Q=10$ scenario at full current and field, i.e. $84 \mathrm{MW} \times 5.2 \mathrm{~T} /(3.1 \times 3.1$ $x 6.2 \mathrm{~m})=7.3 \mathrm{MW} \mathrm{T} / \mathrm{m}$. Here and throughout the rest of the paper, we assume an intrinsic contribution to the core radiation fraction of $30 \%$, i.e. without impurity seeding, $P_{\text {sep }}=0.7\left(P_{f u s} / 5+P_{C D}\right)$. Since in our approach, $B /\left(q_{95} A R\right)$ is kept constant, it follows that the allowable $P_{\text {sep }}$ is constant, too. On the other hand, 
in order to stay in $\mathrm{H}$-mode, $\mathrm{P}_{\text {sep }}$ must exceed the power threshold $P_{L H}$ by a fraction $\mathrm{f}_{L H}$, so that, according to [7]

$$
P_{\text {sep }}>f_{L H} P_{L H}=f_{L H} 1.72 n^{0.78} B^{0.77} a^{0.975} R^{0.999} \approx 1.72 f_{L H}(n B)^{0.78} \frac{R^{2}}{A}
$$

where we have decreased the pre-factor by $2 / 2.5$ since this scaling was derived for $D$ discharges and we assume a scaling proportional to $m_{i}^{-1}$. We also note that core radiation was not subtracted in the scaling, so that there is some margin to justify chosing $f_{L H}$ close to 1 . The combination of (4) and (5) gives a window for $P_{\text {sep }}$ that must be finite for any machine design in our stepladder. Due to the scaling of (5) with $B^{0.8} R^{2}$, $P_{\text {sep }}$ will actually not be constant at constant $f_{L H}$ in the stepladder and hence, the window for $P_{\text {sep }}$ decreases and the core radiation fraction will have to be increased in order to fulfill condition (4).

\section{3.) A numerical example}

We now present an example of a stepladder that serves as an illustration for the approach outlined in the previous section. Starting from an FPP that could be attractive as a base load energy supply, we chose a steady-state tokamak with net electrical power around $1 \mathrm{GW}$, and aim for a minimum recirculating electrical power, which we assume to be dominated by $P_{C D}$, i.e. we neglect the power needed for the balance of plant, assuming water cooling and with it, a thermodynamic efficiency of 0.35 (for He cooling, this would be different). The choice of plasma parameters follows the generic route for steady state tokamaks:

Minimizing $P_{C D}$ according to Eqn. (3) means operation at high $\beta_{N}$ and $q_{95}$, which, at constant fusion power and given machine design $(A, B, R)$, allows to increase both parameters together (see (A1)). However, increasing $q_{95}$ will decrease $Q$ (see Eqn. A2), which can be compensated by finding a regime with higher $H$, so that $H$ and $q_{95}$ can be increased together to keep $Q$ constant. We note that these two arguments together give a justification for the use of the figure of merit $\beta_{N} H / q_{95}{ }^{2}$, for simultaneously approaching high $Q$ and $f_{b s}$ at constant fusion power. However, this is strictly true only at constant heating power and for the particular confinement scaling used here, where $\beta_{N}$ does practically not enter into $Q$ (see [8] for an in-depth discussion). The operation at higher $q_{95}$ also has the benefit of reduced disruptivity, and increasing $\beta_{N}$ usually leads to a regime of higher $H$, such as the 'improved H-mode' regime [9]. Noninductive discharges at elevated $q_{95}$ have been shown in several devices [10], [11], [12], but so far only at q-values that would be prohibitive for ignition (due to the high $\beta_{N}$, the machine cannot be made arbitrarily large since the fusion power would increase too much). We hence set a target of $\beta_{N}=3.5, q_{95}=4.5$ and $\mathrm{H}=1.2$, which have been obtained together in improved $\mathrm{H}$-mode discharges, albeit not under fully noninductive conditions yet. Another caveat is that DEMO and FPP will have to be operated with high radiation fraction and with very small or no ELMs. While $\mathrm{H}$-mode operation has been shown to be in principle compatible with both constraints [13], [14], it is impossible to combine, in present day experiments, the different requirements, such as low collisionality and high Greenwald fraction. Hence, the applicability of the regime is subject to further investigations, both theoretical and experimental, to understand e.g. better the confinement improvement with addition of impurities in the high radiation fraction experiments (we do note that it is found that for stiff profiles, the radial location of the additional radiation zone is crucial [15]). Likewise the absence of ELMs with strong edge velocity shear in the QHmode needs to be understood such that it is clear if how it can be achieved in future devices. 
For the absolute value of the density, we note that it should not be much smaller than in the ITER $Q=10$ scenario to have confidence in the divertor solution developed there, although lowering the density will decrease $P_{C D}$. Based on considerations about the density limit in future low collisionality devices [16], we set $\mathrm{f}_{\mathrm{GW}}=1.2$ and aim for $\mathrm{n} \approx 10^{20} \mathrm{~m}^{-3}$. This will also be helpful for coupling the electron and ion temperature profiles since it allows efficient equipartition (see details in Section 4).

Guided by an analysis using the PROCESS systems code [17] that indicate a solution in this range with $\mathrm{P}_{\text {fus }}$ $=3.5 \mathrm{GW}$ and $60 \%$ bootstrap fraction at $\mathrm{B}=6.1 \mathrm{~T}$ and $\mathrm{R}=8.5 \mathrm{~m}$, resulting in $\mathrm{P}_{\mathrm{CD}} \approx 120 \mathrm{MW}$, we chose this as a model point for an FPP. It is clear that this will have to be consolidated by much more in-depth technical and economic assessment in the future and only serves as a reference here. Assuming a thermodynamic efficiency of 0.35 , this plant will generate an electrical power of $1.450 \mathrm{GW}$ (a factor of 1.18 has been assumed to account for the additional heat generated in the blanket, as used in [4]). Assuming a $40 \%$ wall plug efficiency for $\mathrm{P}_{\mathrm{CD}}$, the net electrical output is then of the order of $1 \mathrm{GW}$, and the recirculating power fraction is about $20 \%$. From $q_{95}=4.5$ and assuming the ITER aspect ratio and shape, we get $I_{p}=16.6 \mathrm{MA}$. At $\mathrm{f}_{\mathrm{GW}}=1.2$, the absolute value of the average density is $8.4 \times 10^{19} \mathrm{~m}^{-3}$.

We can now analyse where a DEMO should sit on the stepladder. Assuming we want to generate several $100 \mathrm{MW}$ as proposed by the Stakeholders [2], we can aim for $P_{\text {fus }}=2 \mathrm{GW}$ which would give close to 0.5 GW net electric power at a recirculating power fraction of $35 \%$, which would be unacceptable for an economic FPP, but as outlined in the previous section the credibility of achieving the $20 \%$ mentioned above for the FPP would be quite high. There is even margin for a lower wall plug efficiency in DEMO that would further reduce the electrical power output, but be acceptable if a convincing case can be made that it can reach the required $40 \%$ in an FPP. This means that $B$ and $R$ should be lower by $(3.5 / 2)^{1 / 7}=1.083$, so that DEMO sits at $7.85 \mathrm{~m}$ and $5.6 \mathrm{~T}$, with $\mathrm{I}_{\mathrm{p}}=14 \mathrm{MA}$.

Now, we can also determine how ITER should be operated to demonstrate this scenario. Scaling down the radius by $7.85 / 6.2=1.26$, the fusion power will be around $400 \mathrm{MW}$, and should be operated at $I_{p}=9 \mathrm{MA}$, $B=4.5 \mathrm{~T}$. However, inserting these values into ( $A 2)$ and using $H=1.2$ as above, we get $Q=1.55$, i.e. the additional heating power needed would be almost $260 \mathrm{MW}$, in excess of ITER's capabilities. It follows that to be consistent with the plans for the heating upgrade of ITER, which aim at $120 \mathrm{MW}$, the H-factor should rather be 1.4. While challenging, such $\mathrm{H}$-factors have been achieved in improved $\mathrm{H}$-mode and clearly set a development target for ITER, i.e. along a path where, starting from the $Q=10$ scenario at $q_{95}=3.1, q_{95}$ is increased together with $\beta_{N}$ as long as the resulting decrease in $Q$ can still be compensated by an increase in $H$ or by adding additional power.

Finally, we analyse how the exhaust problem will vary throughout the stepladder (remember that for our assumptions, this will not be similar throughout the set of parameters). For ITER, the scaling used in (5) gives $P_{L H}=60 \mathrm{MW}$, so an unseeded operation at $P_{\text {heat }}=120 \mathrm{MW}+400 / 5 \mathrm{MW}=200 \mathrm{MW}$ will be well above the threshold $\left(f_{L H}=2.4\right)$. On the other hand, from condition $(4)$, the maximum allowable $P_{\text {sep }}$ is $143 \mathrm{MW}$, so that after subtracting $30 \%$ of intrinsic radiation, the remaining $140 \mathrm{MW}$ are close to the maximum and there is almost no need for an additional core radiation. Testing the radiative scenario, however, can be done by adding seed impurities down to $f_{L H}=1$, i.e. $P_{\text {sep }}=60 \mathrm{MW}$ and core radiation fraction $f_{\text {rad, } \text {,ore }}=0.7$ (i.e. $60 \mathrm{MW}$ intrinsic and $80 \mathrm{MW}$ seeded radiation). It also means that a large window of core radiation fraction can be explored in ITER in this scenario.

As expected from the arguments above, this window shifts and becomes narrower in DEMO and FPP. In DEMO, we find $P_{\text {heat }}=120 \mathrm{MW}+2000 / 5 \mathrm{MW}=520 \mathrm{MW}$ and $P_{L H}=114 \mathrm{MW}$, so that operation at maximum allowable $P_{\text {sep }}=143 \mathrm{MW}$ means $\mathrm{f}_{L H}=1.25$ and $\mathrm{f}_{\text {rad,core }}=0.725$ (which can still be tested in ITER). Operating 
$\mathrm{DEMO}$ at $\mathrm{f}_{\mathrm{LH}}=1$ would then correspond to $\mathrm{f}_{\mathrm{rad}, \mathrm{core}}=0.78$. The same exercise for the FPP gives $\mathrm{P}_{\text {heat }}=120 \mathrm{MW}$ $+3500 / 5 \mathrm{MW}=820 \mathrm{MW}$ and $\mathrm{P}_{\mathrm{LH}}=143 \mathrm{MW}$ so that the operational window shrinks to a point at $\mathrm{f}_{\mathrm{LH}}=1$ and $f_{\text {rad,core }}=0.825$, which means that it has to be assessed in further work what the margins in $P_{\text {sep }}$ and $P_{L H}$ are, and the operational point may have to be slightly adjusted. Fig. 1 shows the margins in $P_{\text {sep, }}, f_{\text {rad,core }}$ and $f_{L H}$ as function of $R$ throughout the stepladder. We note that in reality, the divertor limit will be different in L-mode due to the different $\lambda_{q}$, but since we aim at $\mathrm{H}$-mode operation anyway, this is not taken into account in the diagram.
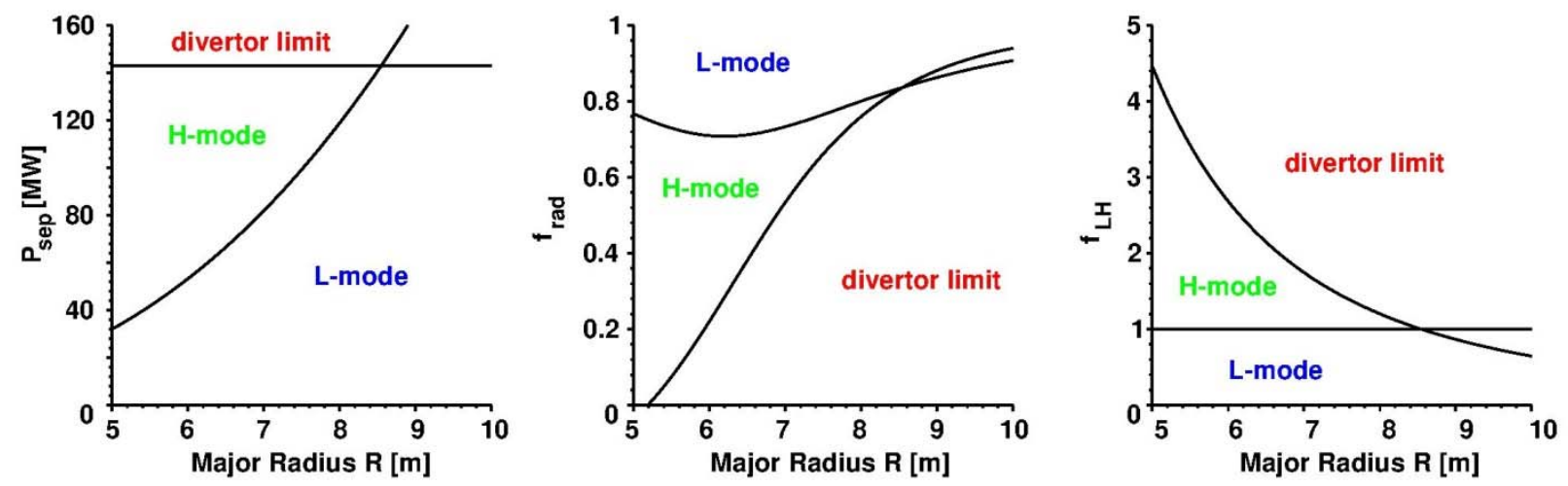

Fig. 1: Narrowing of operational window for $P_{\text {sep, }} f_{\text {rad,core }}$ and $f_{L H}$ : as $R$ increases, the H-mode operational space narrows until at $R=8.5 \mathrm{~m}$ (i.e. the FPP), just one operational point remains.

Finally, table 1 summarises the assumptions and results presented in this section by showing the values of the similarity parameters that have been kept constant on the left hand side and the variation of machine design parameters on the right hand side.

\begin{tabular}{|l|c|c|c|}
\hline & ITER & DEMO & FPP \\
\hline $\mathrm{q}_{95}$ & 4.5 & 4.5 & 4.5 \\
\hline $\mathrm{f}_{\mathrm{GW}}$ & 1.2 & 1.2 & 1.2 \\
\hline $\mathrm{n}_{\mathrm{e}, 19}$ & 8.4 & 8.4 & 8.4 \\
\hline$\beta_{\mathrm{N}}$ & 3.5 & 3.5 & 3.5 \\
\hline $\mathrm{H}$ & 1.4 & 1.2 & 1.2 \\
\hline $\mathrm{f}_{\mathrm{bs}}$ & 0.62 & 0.62 & 0.62 \\
\hline $\mathrm{P}_{\text {sep }} \mathrm{B} /(\mathrm{qAR})$ & 7.3 & 7.3 & 7.3 \\
\hline
\end{tabular}

\begin{tabular}{|l|c|c|c|}
\hline & ITER & DEMO & FPP \\
\hline$R$ & 6.2 & 7.85 & 8.5 \\
\hline $\mathrm{B}$ & 4.5 & 5.6 & 6.1 \\
\hline $\mathrm{I}_{\mathrm{p}}$ & 9 & 14 & 16.6 \\
\hline $\mathrm{P}_{\text {fus }}$ & 0.4 & 2 & 3.5 \\
\hline $\mathrm{P}_{\mathrm{CD}}$ & 0.1 & 0.115 & 0.12 \\
\hline $\mathrm{P}_{\text {el, net }}$ & $\mathrm{n} / \mathrm{a}$ & 0.5 & 1 \\
\hline $\mathrm{f}_{\mathrm{LH}}$ & $1-2.4$ & $1-1.25$ & 1 \\
\hline $\mathrm{f}_{\text {rad,core }}$ & $0.3-0.7$ & $0.72-0.78$ & 0.825 \\
\hline $\mathrm{Q}$ & 3.3 & 17.4 & 29.2 \\
\hline
\end{tabular}

Table 1: Similarity parameters on the stepladder (left) and resulting design parameters (right). For the parameters $f_{L H}$ and $f_{\text {rad,core, }}$ the ranges that can be explored in ITER and DEMO are indicated.

\section{4.) 1.5-D ASTRA modelling}

We have modelled the plasma scenario using the 1.5-D time dependent ASTRA code [18]. The kinetic profiles employ a pedestal with prescribed width of $\Delta \rho=0.06$, where $\rho=r / a$ is the normalized minor radius. The temperature profiles are continued via a simple transport model that assumes the electron and ion heat conductivities to be equal and proportional to $\left(1+\rho^{3}\right)$. The absolute value of energy transport is 
adjusted such that the target $\mathrm{H}$-factor from the 0-D assumption is met, with the chosen value of $H=1.2$ being typical of the 'improved H-mode' scenario [9]. The shape of the electron density profile for $\rho<0.94$ comes from a diffusion coefficient that is proportional to the heat conductivity, together with an inward pinch for particles of the form $v_{i n}=D / R\left(c_{1} R / L_{T}+c_{2} S\right)$ where $L_{T}$ is the temperature scale length and $s$ is the magnetic shear. The constants are chosen to match the theoretical expectations based on TGLF [19]. The particle source is modeled by a Gaussian profile at $\rho=0.9$, in line with expectations for pellet fueling in future devices. The pedestal top density is then calculated from the constraint that the line-averaged density matches the prescribed value. Since the resulting density profiles are quite peaked, the lineaveraged density values exceed the Greenwald-Limit, but the pedestal top stays below, consistent with the approach outlined in [16]. The profiles of all other particles species are derived from specifying a radially constant impurity concentration $f_{Z}=n_{Z} / n_{e}$ for all impurities assumed to be present in the plasma and inferring the resulting $\mathrm{D} / \mathrm{T}$ concentration from quasi-neutrality (an exact 50:50 ratio is assumed). Fig. 2 shows typical kinetic profiles for the three devices in the stepladder.
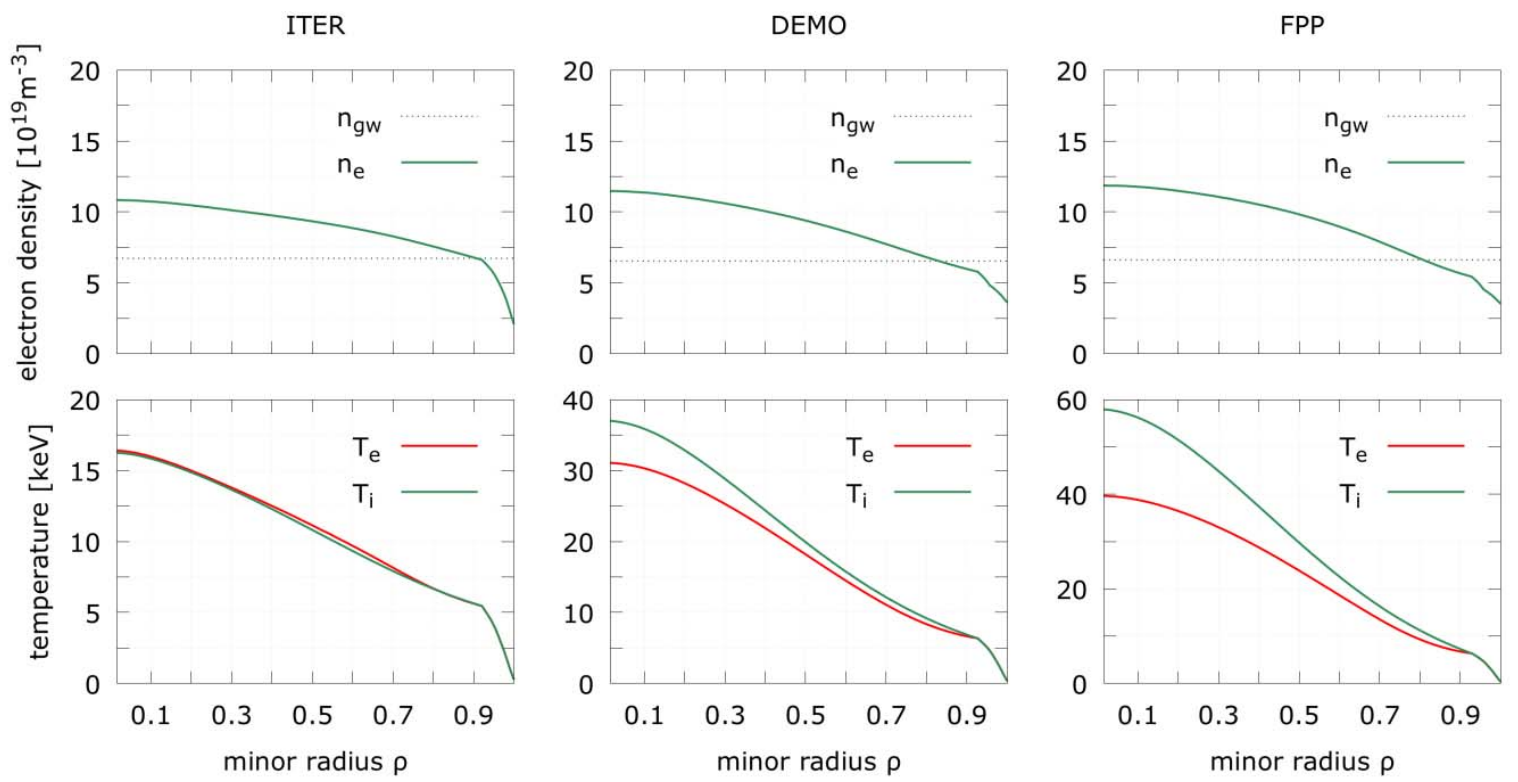

Fig. 2: Kinetic profiles from ASTRA for ITER, DEMO and the FPP.

Interestingly, while for ITER, $T_{e} \geq T_{i}$ in the central region, in line with previous simulations, this trend reverses for DEMO and FPP, with $T_{i}>T_{e}$ being quite pronounced for the latter. This is due to a combination of two effects: on the one hand, the fraction of ion heating increases with $T_{e}$, on the other hand, the increasing radiation losses by impurity seeding enter into the electron heat flux balance. The latter comes from addition of Xenon that is assumed to be injected by a gas valve, i.e. modeled by a very peripheral source in ASTRA and radiation is calculated using the corona loss power function. The concentration $f_{X e}$ is adjusted to match the prescribed $P_{\text {sep }}$ and the numerical values used for ITER/DEMO/FPP are $\left(2.8 \times 10^{-4}\right.$, $\left.6.4 \times 10^{-4}, 7.4 \times 10^{-4}\right)$. The $\alpha$-particles generated in the fusion reaction are the source for a thermal Heconcentration that is fixed to values consistent with $\tau_{\mathrm{He}} / \tau_{\mathrm{E}} \approx 5$. The numerical values used are $f_{\mathrm{He}}=n_{\mathrm{He}} / n_{e}$ $=(0.05,0.08,0.12)$ for (ITER, DEMO, FPP).

Table 2 shows a comparison of integral parameters between the 0-D and the 1.5-D approach. As can be seen, the $0-D$ stepladder can be reproduced, but the major radius, field and current for DEMO and the FPP had to be slightly increased, mainly due to the fact that the high temperatures reached in these devices lead to a weaker than quadratic scaling of $\mathrm{P}_{\text {fus }}$ with $\mathrm{T}_{\mathrm{i}}$. There is a good match in bootstrap fraction 
and total $\beta_{N}$, with the typical thermal $\beta_{N}$-values being of the order of 3.0 (the table quotes the total $\beta$ values). We also note that, as expected, FPP is close to the LH threshold, but the value given in the table is calculated subtracting the total radiation, while the scaling used has an intrinsic radiation of about $30 \%$ that was not corrected for. Using $P_{\text {sepLH }}=0.7 P_{\text {totLH }}$ brings this value to $f_{L H}=1.25$, indicating sufficient margin. Finally, the ITER target could be reached with $\mathrm{H}=1.3$ instead of $\mathrm{H}=1.4$ in the 0 -D approach, relaxing slightly the optimistic assumption that had to be made.

\begin{tabular}{|c|c|c|c|c|c|c|}
\hline \multirow{2}{*}{} & \multicolumn{2}{|c|}{ ITER } & \multicolumn{2}{c|}{ DEMO } & \multicolumn{2}{c|}{ FPP } \\
\cline { 2 - 7 } & ASTRA & 0-D Ansatz & ASTRA & 0-D Ansatz & ASTRA & 0-D Ansatz \\
\hline $\mathrm{P}_{\text {fus }}[\mathrm{MW}]$ & 380 & 400 & 1940 & 2000 & 3480 & 3500 \\
\hline $\mathrm{R}[\mathrm{m}]$ & 6.2 & 6.2 & 8.09 & 7.85 & 9.28 & 8.5 \\
\hline $\mathrm{a}[\mathrm{m}]$ & 2.066 & 2.066 & 2.695 & 2.616 & 3.089 & 2.833 \\
\hline $\mathrm{B}[\mathrm{T}]$ & 4.5 & 4.5 & 5.77 & 5.6 & 6.66 & 6.1 \\
\hline $\mathrm{I}_{\mathrm{p}}[\mathrm{MA}]$ & 9 & 9 & 14.85 & 14 & 19.8 & 16.6 \\
\hline $\mathrm{H}$ & 1.3 & 1.4 & 1.2 & 1.2 & 1.2 & 1.2 \\
\hline$\beta_{\mathrm{N}}$ & 3.07 & 3.5 & 3.4 & 3.5 & 3.59 & 3.5 \\
\hline $\mathrm{q}_{95}$ & 4.82 & 4.5 & 4.81 & 4.5 & 4.9 & 4.5 \\
\hline $\mathrm{f}_{\mathrm{bs}}$ & 0.624 & 0.62 & 0.62 & 0.62 & 0.621 & 0.62 \\
\hline $\mathrm{f}_{\text {rad,core }}$ & 0.525 & $0.3-0.7$ & 0.717 & $0.72-0.78$ & 0.826 & 0.825 \\
\hline $\mathrm{Q}$ & 3.8 & 3.3 & 16.8 & 17.4 & 29 & 29.2 \\
\hline $\mathrm{f}_{\mathrm{LH}}$ & 1.39 & $1-2.4$ & 1.17 & $1-1.25$ & 0.787 & 1 \\
\hline
\end{tabular}

Table 2: Comparison between 0-D Ansatz and 1.5-D ASTRA results for the stepladder example.

Concerning the current and q-profiles, Fig. 3 shows the results for the three devices. It can clearly be seen that the profiles are quite similar, verifying the idea of a common operational scenario. In the simulations, we have split the CD power 50:50 between NBCD and ECCD, with NBCD providing a broad contribution while ECCD is used around the mid-radius to tailor the current profile in detail. This is an optimization process since the ECCD efficiency drops when the deposition is moved to larger radius, mainly because due to the lower temperature, but on the other hand, the q-profile is elevated, resulting in a higher bootstrap fraction. In the example shown in Fig. 3, ECCD was deposited at 4 locations, $\rho_{\text {dep }}=(0.4,0.5,0.6$, $0.65)$ with the ECCD power distributed as a Gaussian with width $\Delta \rho=0.1$ over these radii as $(10 \%, 20 \%$, $30 \%, 40 \%$ ). The total power was set to $100 \mathrm{MW}$ in ITER, $115 \mathrm{MW}$ in DEMO and $120 \mathrm{MW}$ in FPP.

The critical parameter for the $C D$ systems is then the required $C D$ efficiency

$$
\gamma_{C D, 15}=\frac{I_{C D} R n_{e}}{P_{C D}} \frac{15}{T_{e}}
$$

where we have explicitly introduced the temperature dependence and normalized it to $15 \mathrm{keV}$ to be able to compare to previous studies for this temperature range [20]. 



Fig. 3: Modelled current and q-profiles for the three devices.

In our study, we fixed the total power and the NBCD efficiency $\left(\gamma_{15}=0.3\right.$ for ITER and $\gamma_{15}=0.45$ for DEMO and FPP, where the values are normalized to $15 \mathrm{keV}$ and in agreement with [20]), and varied the ECCD efficiency to obtain steady state. This procedure makes sure the operational point does not change when changing the ECCD driven current and the resulting $\gamma_{15, E C C D}$ can then be evaluated. For the power level indicated above, we obtain $\gamma_{15, E C C D}=0.23$ for ITER, $\gamma_{15, E C C D}=0.165$ for DEMO and $\gamma_{15, E C C D}=0.27$ for FPP. Comparing to [20], these values should be within reach, and an analysis using the TORBEAM code for the DEMO operational point from ASTRA in fact yields normalized $\gamma_{C D, 15}$ of the order 0.15-0.2 for ECCD at 230 $\mathrm{GHz}$.

We have also studied the option of a 'flexi-DEMO', i.e. the use of our DEMO device as designed with ASTRA as a pulsed tokamak in case the envisaged scenario turns out to be too optimistic. Table 3 shows the result of a scan in $\mathrm{H}$-factor - reducing $\mathrm{H}$ leads to a loss in confinement which can be recovered by increasing the current and density (at constant $f_{G W}$ ), but will, for given $C D$ power, lead to a pulsed operation. It can be seen from Table 3 that in this scan, also $\beta_{N}$ decreases with $H$, so that the operational point will move further away from the ideal MHD $\beta$-limit, so correspond to more conservative physics assumptions also in this respect. 


\begin{tabular}{|c|c|c|c|c|c|c|}
\hline H-factor & $\mathrm{I}_{\mathrm{p}}[\mathrm{MA}]$ & $\mathrm{n}_{\mathrm{e}, 19}$ & $\mathrm{I}_{\text {ohm }}[\mathrm{MA}]$ & $\mathrm{U}_{\text {ind }}[\mathrm{mV}]$ & $\beta_{\mathrm{N}, \text { therm }}$ & $\tau_{\text {pulse }}[\mathrm{h}]$ \\
\hline 1.2 & 14.85 & 7.81 & 0 & 0 & 2.99 & s.s. \\
\hline 1.15 & 15.15 & 7.97 & 1.17 & 3.65 & 2.89 & 10.26 \\
\hline 1.1 & 15.55 & 8.18 & 2.19 & 7.38 & 2.8 & 4.94 \\
\hline 1.05 & 16 & 8.41 & 3.34 & 12.58 & 2.7 & 2.81 \\
\hline 1 & 16.45 & 8.65 & 4.59 & 17.02 & 2.6 & 2.02 \\
\hline 0.95 & 16.8 & 8.83 & 5.77 & 22.76 & 2.46 & 1.47 \\
\hline
\end{tabular}

Table 3: pulse length in DEMO for decreasing $\mathrm{H}$-factor.

This trend has been verified using the MARS-F ideal MHD stability code [21], [22], to find the $\beta_{\mathrm{N}}$-value at which the plasma becomes unstable to $\mathrm{n}=1$ kink modes. For the stepladder DEMO operating at $\beta_{N, \text { tot }}=3.5$, we find that the operational point is far above the no-wall limit $\left(\beta_{N, n o-w a l l}=2\right.$ for this case, see Fig. 4 left $)$ ), but below the ideal wall limit for reasonable distance of the wall: at $r_{\text {wall } a}=1.2$, the $\mathrm{n}=1$ mode becomes unstable at $\beta_{N}=5.2$. However, since the effect of the stabilizing wall becomes less pronounced for higher $\mathrm{n}$-modes, the actual $\beta$-limit is set by the $\mathrm{n}=2$ mode at $\beta_{N}=4.2$, still well above the operational point (right part of Fig. 4).
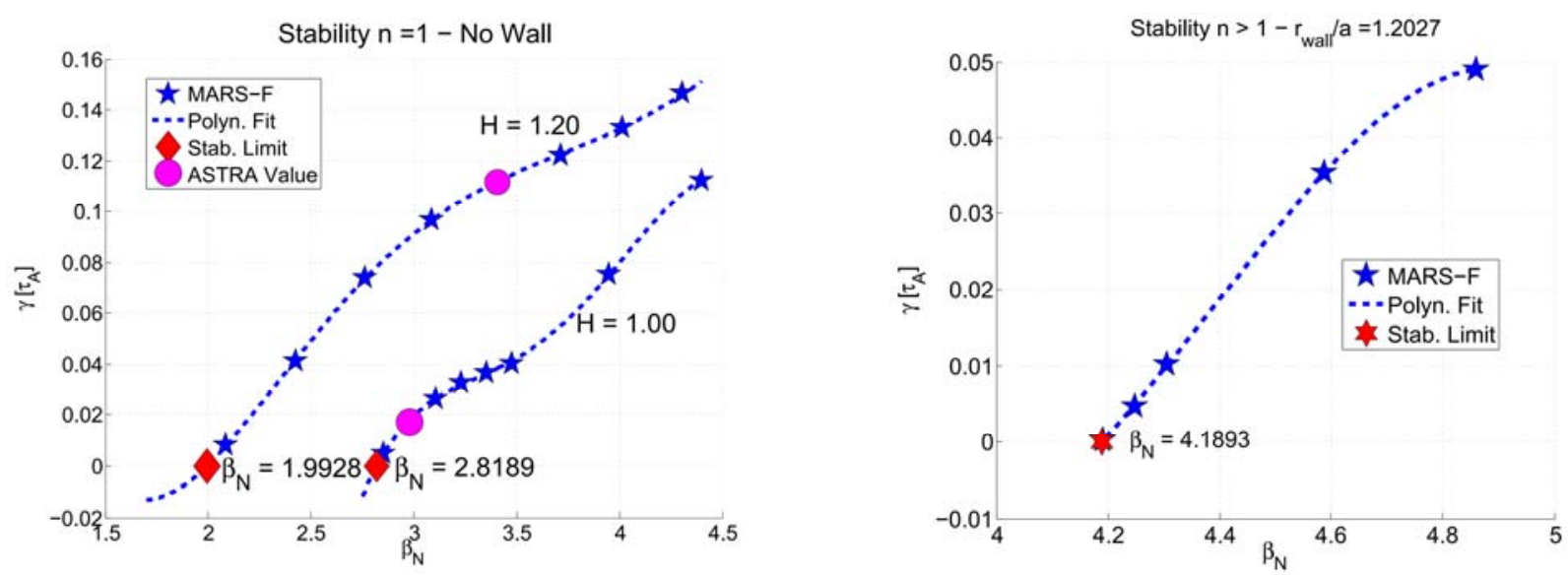

Fig. 4: Results of ideal MHD stability analysis: the no-wall limit, set by $n=1$ (left part), for the DEMO design from the stepladder $\left(H=1.2, \beta_{N, \text { tot }}=3.5\right)$ is found to be $\beta_{N, \text { tot }}=2$, indicating the need for a stabilizing wall. The flexi-DEMO at $H=1, \beta_{N, \text { tot }}=2.9$ is close to the no-wall limit at $\beta_{N, t o t}=2.8$. Adding an ideal wall at $r_{\text {wall }} / a=1.2$, the limit is set by the $n=2$ mode at $\beta_{N}=4.2$ (right part).

Such a low no-wall limit and a large benefit of a stabilising wall is typical for elevated q-profiles [23] and indicates that the plasma may be unstable to Resistive Wall Modes (RWMs). These may be stabilized by either the combined effect of rotation and kinetic effects from both thermal and fast particles or using active control, which have not been considered here (in our analysis, $\beta_{N, \text { tot }}$ has been calculated as the sum of thermal and fast particle pressure, but kinetic effects are not included). A detailed study is however beyond the scope of this paper. Reducing $\beta_{N}$ in the flexi-DEMO, we find that the case with $\mathrm{H}=1, \beta_{N, \text { therm }}=$ 2.6 from Table 3 is close to the no-wall stability, since MARS-F indicates a no-wall limit of $\beta_{N, n o-w a l l}=2.8$ and the total $\beta_{N}$ is 2.95 . 
Since ASTRA allows to obtain the loop voltage for these cases, but does not know about the tokamak coil system and available poloidal flux, the pulse lengths listed in the table have been estimated using simple scaling relations assuming that the maximum field in the $\mathrm{OH}$ coil is limited to the ITER value and the coil sizes increase linearly with major radius. These calculations have been cross-checked using PROCESS, which uses a consistent coil set, and reasonable agreement is found between the two methods, as shown in Fig. 5 (the differences in current are mainly due to profile effects). As can be seen, at $\mathrm{H}=1.0$, this device would still provide a pulse length of about $2 \mathrm{hrs}$, in line with the EU conservative DEMO design [2].


Fig. 5: Plasma current and pulse length as a function of $\mathrm{H}$-factor from PROCESS mimicking the scan with ASTRA shown in Table 3.

\section{5.) Discussion and conclusions}

We have presented an approach to design in a consistent way a stepladder connecting ITER, DEMO and an FPP. This approach starts from an attractive FPP and then locates DEMO such that it will produce considerably less power (making the plant smaller and hence less expensive), but since main similarity parameters for the core scenario are constant, allows a confident extrapolation to an FPP, as is required for a single-step approach from DEMO to a commercial FPP. Furthermore, the approach presented suggests how to use ITER such that DEMO can be extrapolated with maximum confidence. In essence, the approach allows to verify key physics elements in ITER such that DEMO is mainly a technological demonstrator and not a plasma physics experiment. Interestingly, the proposed ITER operation scenario is closer to the $Q=5$ steady state scenario than to the $Q=10$ pulsed operation, which shows the important role of ITER in scenario development, in addition to reaching the $Q=10$ goal. A development path for plasma scenarios in ITER follows from our approach, moving from low $\beta_{N}$ and $q_{95}$ typical for the present $Q=10$ scenario to higher values needed for steady state. We note that the fraction of $\alpha$-heating will increase throughout the stepladder, while the physics basis used in our approach is based on machines with dominant external heating. It will be important to understand how the effects of dominant self-heating, and the stepladder may be refined as better understanding emerges, very importantly also through ITER.

A numerical example has been given, indicative of the feasibility of the approach, and it is backed up by more detailed 1.5-D calculation using the ASTRA code. We note that ideal MHD stability analysis of the DEMO 
operating point indicates that it is located between the no-wall and the ideal wall $\beta$-limit, which may require active stabilization. While certainly being subject to further optimization in future work, e.g. when further advances in plasma physics give rise to more optimistic assumptions, it serves as an example of a stepladder that fulfills the requirements for an FPP and describes where to localize DEMO on this stepladder. We note here that the DEMO point could be scaled up or down in different development strategies, i.e. moved closer to the FPP if efficient generation of electricity would already be deemed important for DEMO or moved closer to ITER if this should be de-emphasized, e.g. in an approach that targets a pilot plant demonstrating electrical self-sufficiency without actually producing electricity [24]. We note that in our proposed approach, the a-power is 3.5 times the external heating power, moving the plasma much closer to the self-organised state typical for a reactor than in the ITER $Q=10$ scenario where this ratio is 2 .

We note here that in the proposed approach, DEMO is of a moderately advanced type (steady state using an 'improved H-mode type' scenario), but the basic features are not too different from the more conservative present EU design such that it could still be used as a pulsed fallback solution should a stationary operation turn out to be impossible. We have shown that, for a reduction of $\mathrm{H}$-factor by $20 \%$, the pulse length would still be around $2 \mathrm{hrs}$. Conversely, finding regimes of further improved plasma stability and confinement may increase the bootstrap fraction, which in turn would lead to higher Q operation and make the FPP more attractive.

Finally, while the present approach targets the plasma scenario, a similar exercise should be conducted concerning technology developments, again with the aim to demonstrate the necessary technology in DEMO such that the step to the FPP becomes credible. For example, one might conceive building DEMO for a higher maximum B-field than needed in the similarity scenario if this is deemed necessary for demonstrating the magnet technology (such a step would also increase the flexibility of the device for adjusting the scenario). Examining the constraints set by technology can also give an indication of the possible gain if some of the margins can be increased. For example, the use of advanced divertor concepts, which may allow to increase the permissible power flux across the separatrix, could reduce the required core radiation fraction.

One of the authors (M.S.) would like to thank Dr. Y. Liu (CCFE Culham) for his support in carrying out the MARS simulations. This work has been carried out within the framework of the EUROfusion Consortium and has received funding from the Euratom research and training programme 2014-2018 under grant agreement number 633053. The views and opinions expressed herein do not reflect those of the European Commission. 


\section{Appendix: 0-D relations to assess the fusion performance of a tokamak}

Similar to the approach used in [3], we start from the equation for the fusion power

$$
P_{f u s}=\frac{c_{1} \cdot \beta_{N}^{2} B^{4} R^{3}}{A^{4} q_{95}^{2}}
$$

where the machine size is described major radius $R$ and aspect ratio $A$, and $B$ is the toroidal magnetic field. The plasma performance is described in dimensionless plasma parameters, namely normalized pressure $\beta_{N}$, and safety factor $q_{95}$. The latter is related to the cylindrical safety factor $q_{c y l}$ by the shaping factor $S$ :

$$
q_{95}=S q_{c y l} \text { with } q_{c y l}=5 R B_{t} /\left(I_{p} A^{2}\right)
$$

where $I_{p}$ is given in units of MA. Note that $c_{1}$ will depend on the shape of the poloidal cross-section through $S$, which is assumed to be fixed here.

The energy amplification $Q=P_{f u s} / P_{A U X}$ is given by

$$
Q=\frac{1}{\frac{c_{2}}{c_{1}} \frac{q_{95}^{3.1} A^{3.53}}{H^{3.23} \beta_{N}{ }^{0.1} B^{3.7} R^{2.7}}-\frac{1}{5}}
$$

where the ITER98 $(\mathrm{p}, \mathrm{y}) \mathrm{H}$-mode confinement scaling has been assumed and $H=\tau_{E} / \tau_{98}$ is the confinement enhancement factor. In (A1) and (A3), the normalised $\beta_{N}$ has been used since it is the quantity related to ideal MHD stability limits. It can be expressed through the total $\beta$ by

$$
\beta_{N}=c_{3} A q_{95} \beta
$$

and the poloidal beta, $\beta_{p}$, is given by

$$
\beta_{p}=c_{4}\left(A q_{95}\right)^{2} \beta
$$

Different from [3], where the temperature has been assumed to be constant and hence the density implicitly varied, we now explicitly prescribe the volume averaged density $n$ such that the volume averaged temperature $T$ follows from the total $\beta$ through

$$
\beta=c_{5} \cdot \frac{n T}{B^{2}}
$$

The density is expressed in terms of the Greenwald density $n_{G W}=I_{p} /\left(\pi a^{2}\right)$ by the Greenwald fraction $f_{G W}$

$$
n=f_{G W} \cdot n_{G W}=f_{G W} \cdot \frac{I_{p} A^{2}}{\pi R^{2}}
$$

Another shortcoming of [3] was that no account was made of the power exhaust problem, which limits the power crossing the separatrix, $P_{\text {sep }}$ 


$$
P_{\text {sep }}=\frac{P_{\text {fus }}}{5}+P_{C D}-P_{\text {rad }}=\left(\frac{P_{\text {fus }}}{5}+P_{C D}\right)\left(1-f_{\text {rad,core }}\right)
$$

where the radiated power in an impurity seeded scenario can crudely be parametrised by

$$
P_{\text {rad }}=c_{6} \cdot n^{2} Z_{\text {eff }} \frac{R^{3}}{A^{2}}
$$

noting that there will also be an 'intrinsic' radiation loss due to Bremsstrahlung and synchrotron losses that is not accounted for in (A8) and the constant $c_{6}$ will strongly depend on the seed impurity and also on the temperature.

Finally, we parametrize the $P_{C D}$, the power needed to obtain steady state tokamak conditions, i.e. to drive the part of the plasma current $I_{p}$

$$
I_{p}=c_{7} \cdot \frac{R}{A^{2}} \frac{B}{q_{95}}
$$

that is not intrinsically driven by the bootstrap current, by

$$
P_{C D}=c_{8}\left(5+Z_{e f f}\right) I_{p} \frac{n}{T} R\left(1-c_{9} \cdot \frac{1}{\sqrt{A}} \beta_{p}\right)
$$

where the last bracket reduces $I_{p}$ by the bootstrap current and the factor $5+Z_{\text {eff }}$ is for RFCD schemes, while for NBCD, the $Z_{\text {eff }}$ dependence is rather of the form $\left(1-Z_{\text {beam }} / Z_{\text {eff }}+f\left(A, Z_{\text {beam }} / Z_{\text {eff }}\right)\right)$ where the function $f\left(A, Z_{\text {beam }} / Z_{\text {eff }}\right)$ can be found e.g. in [25].

For constant shape, the parameters $c 1, c 2, c 3, c 4, c 5$ and $c 7$ can be determined and, for ITER shape, are given by

$$
c_{1}=0.629, c_{2}=6.79, c_{3}=0.104, c_{4}=0.00331, c_{5}=8.04, c_{7}=13.85
$$

The constants $c_{8}$ and $c_{9}$ can be derived from a steady state DEMO simulation using PROCESS to be

$$
c_{8}=5.38, c_{9}=0.7
$$

and finally, for the same DEMO run employing Xe as seed impurity, we get

$$
\mathrm{C}_{6}=19.5
$$

This set of parameters reproduces well fusion power, bootstrap fraction and current drive power for the particular PROCESS run. 


\section{References}

[1] K. Lackner, J. Nucl. Mater. 307 (2002) 10.

[2] G.F. Federici et al., Fus. Eng. Des. 89 (2014) 882.

[3] D. Maisonnier et al., Nucl. Fusion 47 (2007) 1524.

[4] H. Zohm, Fus. Sci. Technology 58 (2010) 613.

[5] B.F. Mc Millan et al., Phys. Rev. Lett 105 (2010), 155001.

[6] T. Eich et al., Phys. Rev. Lett. 107 (2011) 215001.

[7] Y. Martin et al., Journal of Physics: Conference Series 123 (2008) 012033.

[8] A.G. Peeters et al., Nucl. Fusion 47 (2007) 1341.

[9] A.C.C. Sips et al., Plasma Phys. Control. Fusion 44 (2002) B69.

[10] F. Turco et al., Phys. Plasmas 22 (2015) 056113.

[11] A. Garofalo et al., Nucl Fusion 55 (2015) 123025.

[12] A. Bock et al., 43 ${ }^{\text {rd }}$ EPS Conference on Plasma Physics, Leuven, Belgium, (2016) 03.110.

[13] A. Kallenbach et al., Nucl. Fusion 52 (2012) 122003.

[14] K.H. Burrell et al., Phys. Plasmas 23 (2016) 056103.

[15] E. Fable et al., Nucl. Fusion

[16] H. Zohm et al., Nucl. Fusion 53 (2013) 073019.

[17] M. Kovari et al., Fus. Eng. Des. 89 (2014) 3054.

[18] G. V. Pereverzev et al., IPP Report 5/42 (1991).

[19] G. Staebler et al., 2005 Phys. Plasmas 12 (2005) 102508.

[20] H. Zohm et al., 40 ${ }^{\text {th }}$ EPS Conference on Plasma Physics, Helsinki, Finland, ECA 37D (2013) 03.108.

[21] Y. Liu et al., Phys. Plasmas 15, 112503 (2008),

[22] Y. Liu et al., Phys. Plasmas 21, 056105 (2014)

[23] J. Manickam et al., Phys. Plasmas 1 (1994) 1601.

[24] J. Menard et al., Nucl. Fusion 51 (2011) 103014.

[25] J.W. Connor et al., Nucl. Fusion 14 (1974) 185. 